\title{
Antibiotic Use and Need for Antimicrobial Stewardship in Long-Term Care
}

\author{
Lisa Dong-Ying Wu, Sandra A N Walker, Marion Elligsen, Lesley Palmay, Andrew Simor, \\ and Nick Daneman
}

\section{ABSTRACT}

Background: Antimicrobial stewardship may be important in long-term care facilities because of unnecessary or inappropriate antibiotic use observed in these residents, coupled with their increased vulnerability to health care-associated infections.

Objectives: To assess antibiotic use in a long-term care facility in order to identify potential antimicrobial stewardship needs.

Methods: A retrospective descriptive study was conducted at the Veterans Centre, a long-term care facility at Sunnybrook Health Sciences Centre, Toronto, Ontario. All residents taking one or more antibiotics $(n=326)$ were included as participants. Antibiotic-use data for patients residing in the facility between April 1, 2011, and March 31, 2012, were collected and analyzed.

Results: Totals of 358 patient encounters, 835 antibiotic prescriptions, and 193 positive culture results were documented during the study period. For 36\% (302/835) of antibiotic prescriptions, the duration was more than 7 days. Cephalosporins $(30 \% ; 251 / 835)$ and fluoroquinolones $(28 \% ; 235 / 835)$ were the most frequently prescribed antibiotic classes. Urine was the most common source of samples for culture $(60 \%$; 116/193).

Conclusions: Characteristics of antimicrobial use at this long-term care facility that might benefit from further evaluation included potentially excessive use of fluoroquinolones and cephalosporins and potentially excessive duration of antibiotic use for individual patients.

Keywords: antimicrobial stewardship, long-term care, antibiotic use

Can J Hosp Pharm. 2015;68(6):445-9

\section{RÉSUMÉ}

Contexte : La gérance des antibiotiques peut s'avérer importante au sein des établissements de soins de longue durée à cause d'une utilisation inutile ou inappropriée des antibiotiques chez les résidents de ces établissements et de leur vulnérabilité aux infections nosocomiales.

Objectifs : Évaluer l'utilisation des antibiotiques dans un établissement de soins de longue durée afin de déterminer si une gérance des antimicrobiens peut être nécessaire.

Méthodes : Une étude descriptive rétrospective a été réalisée au Veterans Centre, un établissement de soins de longue durée au sein du Sunnybrook Health Sciences Centre, à Toronto en Ontario. Tous les résidents prenant au moins un antibiotique $(n=326)$ ont été admis à l'étude. Des données sur les antibiothérapies pour des patients résidant dans l'établissement entre le 1er avril 2011 et le 31 mars 2012 ont été recueillies et analysées.

Résultats : Pendant l'étude, on a consigné en tout 358 séjours de patients, 835 prescriptions d'antibiotiques et 193 résultats positifs de culture. Pour $36 \%$ (302/835) des prescriptions d'antibiotiques, le traitement était de plus de 7 jours. Les céphalosporines (30\% [251/835]) et les fluoroquinolones (28\% [235/835]) étaient les antibiotiques les plus souvent prescrits. Les cultures étaient le plus souvent obtenues à partir d'urines (60\% [116/193]).

Conclusions : L'utilisation possiblement excessive de fluoroquinolones et de céphalosporines ainsi que la durée potentiellement exagérée des antibiothérapies font partie des caractéristiques de l'emploi des antimicrobiens dans cet établissement de soins de longue durée qui pourraient mériter de plus amples évaluations.

Mots clés : gérance des antimicrobiens, soins de longue durée, antibiothérapie 


\section{INTRODUCTION}

$\mathrm{A}$ ntimicrobial stewardship may be important in long-term care facilities because residents in these facilities are especially vulnerable to health care-associated infections, as a result of functional impairment, declines in their immune systems, and the care environment. ${ }^{1}$ Similar to the situation in acute care hospital settings, ${ }^{2,3}$ it is estimated that about half of antibiotic use in long-term care facilities is unnecessary or inappropriate. ${ }^{4}$ Consequently, there is great concern about adverse reactions and selection of antibiotic-resistant pathogens ${ }^{5}$ in this setting. In addition, the development of new antimicrobial agents has slowed, ${ }^{6}$ leaving clinicians with few options to treat antibioticresistant infections, which are associated with increases in length of stay, mortality, and cost. ${ }^{7}$

Evidence from a recent systematic review, ${ }^{8}$ a controlled interrupted time series analysis of antimicrobial stewardship in critical care, ${ }^{9}$ and a randomized study with stepped wedge design involving ward patients at Sunnybrook Health Sciences Centre, Toronto, Ontario, ${ }^{10}$ has shown that antimicrobial stewardship programs were associated with reductions in antibiotic use, costs, adverse events, and antimicrobial resistance without compromising clinical outcomes such as nosocomial infection rates, length of stay, or mortality rates among critically ill and ward patients in acute care settings.

However, few studies have focused on antimicrobial stewardship for residents living in long-term care facilities. ${ }^{11,12}$ Therefore, identification of unique antimicrobial stewardship strategies to optimize antibiotic use in such facilities is greatly needed.

The objective of this study was to assess antibiotic use in the Sunnybrook Health Sciences Centre - Veterans Centre (SHSCVC) long-term care facility, with the ultimate goal of determining potential antimicrobial stewardship needs.

\section{METHODS}

\section{Study Design, Setting, and Data Source}

This retrospective descriptive study of antibiotic use among long-term care residents was conducted across 17 long-term care units at SHSC-VC in Toronto, Ontario. SHSC-VC is the largest veterans' care facility in Canada, offering long-term and complex care, specifically cognitive support (8 units), mental health and addiction treatment ( 1 unit), physical support (7 units), and palliative care (1 unit). It has a capacity of about 500 inpatient beds, with an approximate average of 13507 patient-days per month at the time of this study. ${ }^{13}$ Potential participants for the current study were identified through the SPIRIT database ${ }^{14}$ of the Antimicrobial Stewardship Program at Sunnybrook Health Sciences Centre.

The Sunnybrook Research Ethics Board originally granted approval on February 4, 2010, for review of the SPIRIT database to identify quality improvement needs for antimicrobial stewardship at Sunnybrook Health Sciences Centre; this ethics approval undergoes annual review.

\section{Patient Eligibility}

All residents living at the SHSC-VC long-term care facility for whom antibiotics were prescribed between April 1, 2011, and March 31, 2012, as identified by the SPIRIT database, were included in this study.

\section{Data Collection}

The following data were retrieved from the SPIRIT database: patient identifiers (hospital file number and encounter number), location, service, date of admission, date of discharge or death, antibiotic(s) administered (medication, subclass, class, dose, frequency), start and stop dates of antibiotic(s), route of therapy, and culture and susceptibility results for a specific source identified by unique accession number. Antibiotic prescriptions were grouped by generic drug names, as well as pharmacological class and subclass, as follows: aminoglycosides, carbapenems, cephalosporins (first-, second-, and third-generation), fluoroquinolones (ciprofloxacin, levofloxacin, moxifloxacin), glycopeptides (oral and IV vancomycin), macrolides, penicillins (amoxicillin, amoxicillin-clavulanate, ampicillin, cloxacillin, penicillin, piperacillin-tazobactam), sulfonamides, tetracyclines, and miscellaneous (clindamycin, metronidazole, nitrofurantoin, rifampin). In addition, the source of each culture sample was identified as follows: blood, urine, wound swab, other swab, other fluid, dialysate, expectorated sputum, or stool.

Duration of therapy was calculated using the start and stop dates of each antibiotic. Each antibiotic order was treated as a distinct prescription, with no overlap or extension in duration of therapy. The duration of antibiotic therapy was classified as shortcourse ( $\leq 7$ days) or long-course ( $>7$ days); in addition, therapy lasting 90 days or more was classified as long-term prophylaxis or long-term therapy.

\section{Outcome Assessment}

The primary outcome of interest was median days of therapy (DOT) per 1000 patient-days per month ${ }^{12}$ from April 1, 2011, to March 31, 2012. Any dose of an antibiotic received during a 24-h period represented 1 DOT. The total DOT for a given patient who was receiving multiple antibiotics was the sum of DOTs for all antibiotics. The secondary outcomes of interest were the proportion of patients treated for more than 7 days; comparisons of antimicrobial classes, specific agents, and routes of administration; antibiotic use in unit wards; culture sources and results of sensitivity testing; and prevalence of positive results on testing for Clostridium difficile (by polymerase chain reaction for toxin B). 


\section{Statistical Analysis}

Descriptive analysis of the data was carried out using Microsoft Excel 2007 software (Microsoft Corporation, Redmond, Washington). DOT values are reported as medians because the antimicrobial-use data were not normally distributed.

\section{RESULTS}

\section{Description of Unit Wards and Demographic Characteristics}

Based on the total of 835 antibiotic orders during the study period, the final patient cohort consisted of 326 residents living in the 17 units in the SHSC-VC. Some residents were admitted more than once to specific facility units, so the total number of distinct patient encounters was 358, consisting of 128 encounters in the physical support units (355 antibiotic orders), 116 in the cognitive support units (287 orders), 102 in the palliative care unit (173 orders), and 12 in the mental health and addiction unit (20 orders). The median age of residents included in this study was 89 years (range 22-100), and most of the patients were men (242; 74\%). The total number of patient-days at SHSC-VC during the study period was 162080 days.

\section{Antibiotic Use}

Overall, 302 (36\%) of the 835 antibiotic prescriptions had a duration of therapy longer than 7 days. Only 2 prescriptions $(0.2 \%)$ had a duration exceeding 90 days, which suggested that long-term treatment and prophylaxis were rare. The median DOT per 1000 patient-days per month was 2.7 (interquartile range $[\mathrm{IQR}] 2.1-3.0)$ for all antibiotic routes combined (IV, oral, gastric tube, intraperitoneal, and topical), 0.48 (IQR 0.41-0.62) for IV antibiotics, and 1.9 (IQR 1.6-2.4) for oral antibiotics. The median number of antibiotic orders per patient encounter was 2 (IQR 1-3) for all long-term care units combined, 2 (IQR 1-4) for the physical support units, 2 (IQR 1-3) for the cognitive support units, 1 (IQR 1-2) for the palliative care unit, and 1 (IQR 1-2) for the mental health and addiction unit.

Ciprofloxacin ( $n=127$ antibiotic orders; $15 \%)$, levofloxacin ( $n=105 ; 13 \%)$, and ceftriaxone $(n=96 ; 11 \%)$ were the most commonly used antibiotics. Cephalosporins $(n=251 ; 30 \%)$ and fluoroquinolones ( $n=235 ; 28 \%$ ) were the most commonly used antibiotic classes, each having a median duration of therapy of 0.043 per 1000 patient-days (Table 1$)$. The oral $(n=594 ; 71 \%)$ and IV ( $n=208 ; 25 \%)$ routes were most commonly prescribed.

\section{Culture Methods and Sensitivity}

Most courses of antibiotic therapy were started and completed without associated positive culture results (193 positive results for 835 antibiotic orders; 23\%). Of the 193 positive culture results, urine was the most frequent source (116;
60\%). Escherichia coli was the most common bacteria isolated from urine ( $45 \% ; 52 / 116)$, and $37 \%$ (43/116) of the patients who had urine samples drawn for culture were treated with ciprofloxacin. Of the patients whose urine samples grew E. coli, 40\% (21/52) were receiving ciprofloxacin, even though $24 \%$ of these (5/21) had ciprofloxacin-resistant $E$. coli. During the study period, a total of 528 urine samples were sent for culture, of which $328(62 \%)$ were negative; concurrent antibiotics were prescribed for 108 (33\%) of these cases with negative results on urine culture.

E. coli was isolated from 68 samples overall (any source), of which $46(68 \%)$ were susceptible to ciprofloxacin. Available alternatives to ciprofloxacin in the setting of either empiric treatment or documented urinary tract infection with resistant E. coli, based on the observed susceptibility profile in the longterm care facility, include nitrofurantoin (E. coli susceptibility 91\% [62/68]) for cystitis and either amoxicillin-clavulanate (E. coli susceptibility $74 \%$ [50/68]; E. coli susceptibility to ampicillin $57 \%$ [39/68]) or trimethoprim-sulfamethoxazole (E. coli susceptibility $74 \%$ [50/68]) for either cystitis or pyelonephritis. The prevalence of positive $C$. difficile tests was $2.4 \%$ (8/326) with a mean duration of therapy for $C$. difficile infection of 10 days.

\section{DISCUSSION}

This descriptive study assessed antibiotic use in the SHSCVC long-term care facility to determine potential antimicrobial stewardship needs. The results of this study generated several hypotheses for more in-depth evaluation to determine whether any antimicrobial stewardship interventions may be of value in the long-term care setting, including prospective audit and feedback for frequently used antibiotic classes (cephalosporins and fluoroquinolones) and prospective audit and feedback for all patients with antibiotic therapy longer than 7 days.

These results lend further support to the limited database of literature concerning the potential need for antimicrobial stewardship in long-term care facilities. Daneman and others ${ }^{11}$ found that $62.6 \%$ of all antibiotic treatment courses for Ontario long-term care residents were at least 10 days in duration, and $20.9 \%$ exceeded 90 days. Similarly, at SHSC-VC, antibiotic therapy was prolonged (longer than 7 days) for $36 \%$ of prescriptions. Daneman and others ${ }^{11}$ and Jump and others ${ }^{12}$ showed that the most common antibiotics (nitrofurantoin, trimethoprimsulfamethoxazole, and ciprofloxacin) used for long-term care residents were indicated for suspected urinary tract infections. In the current study, urine was the most common culture source and ciprofloxacin the most commonly used antibiotic, results that concur with previous studies. ${ }^{11,12}$ A prospective study evaluating the indications for use of antibiotics in one or more long-term care facilities would be of value to definitively determine whether fluoroquinolones are most commonly used to treat suspected urinary tract infections in this patient population. 
Table 1. Overall Antibiotic Use and Duration of Therapy over Study Period*

\begin{tabular}{|c|c|c|c|}
\hline \multirow{2}{*}{$\begin{array}{l}\text { Antibiotic Use } \\
\text { Monthly use, by route }\end{array}$} & \multirow[t]{2}{*}{ No. of Orders } & \multicolumn{2}{|c|}{$\begin{array}{c}\text { Median DOT/1000 Patient-Days } \\
\text { (and IQR) }\end{array}$} \\
\hline & & & \\
\hline Total antibiotics, any route† & 835 & 2.7 & $(2.1-3.0)$ \\
\hline $\mathrm{PO}$ & 594 & 1.9 & $(1.6-2.4)$ \\
\hline IV & 208 & 0.48 & $(0.41-0.62)$ \\
\hline \multicolumn{4}{|l|}{$\begin{array}{l}\text { Total use, by class, subclass, } \\
\text { or individual drug }\end{array}$} \\
\hline Aminoglycosides & 10 & 0.025 & $(0.006-0.029)$ \\
\hline Carbapenems & 2 & 0.059 & $(0.045-0.072)$ \\
\hline Cephalosporins & 251 & 0.043 & $(0.019-0.049)$ \\
\hline First-generation & 81 & 0.043 & $(0.031-0.062)$ \\
\hline Second-generation & 54 & 0.049 & $(0.019-0.062)$ \\
\hline Third-generation & 116 & 0.043 & $(0.019-0.049)$ \\
\hline Clindamycin & 15 & 0.031 & $(0.019-0.049)$ \\
\hline Fluoroquinolones & 235 & 0.043 & $(0.025-0.049)$ \\
\hline Ciprofloxacin & 127 & 0.043 & $(0.025-0.049)$ \\
\hline Levofloxacin & 105 & 0.043 & $(0.031-0.049)$ \\
\hline Moxifloxacin & 3 & 0.049 & $(0.034-0.151)$ \\
\hline Macrolides & 56 & 0.022 & $(0.006-0.025)$ \\
\hline Metronidazole & 43 & 0.049 & $(0.012-0.062)$ \\
\hline Nitrofurantoin & 39 & 0.043 & $(0.043-0.049)$ \\
\hline Penicillins & 103 & 0.043 & $(0.019-0.049)$ \\
\hline Amoxicillin & 49 & 0.043 & $(0.037-0.049)$ \\
\hline Amoxicillin-clavulanate & 7 & 0.062 & $(0.043-0.241)$ \\
\hline Ampicillin & 9 & 0.019 & $(0.006-0.049)$ \\
\hline Cloxacillin & 20 & 0.046 & $(0.035-0.062)$ \\
\hline Penicillin & 1 & 0.006 & $(0.006-0.006)$ \\
\hline Piperacillin-tazobactam & 17 & 0.012 & $(0.006-0.049)$ \\
\hline Rifampin & 4 & 0.043 & $(0.043-0.043)$ \\
\hline Sulfamethoxazole-trimethoprim & 41 & 0.043 & $(0.019-0.049)$ \\
\hline Tetracyclines & 16 & 0.046 & $(0.043-0.062)$ \\
\hline Vancomycin IV & 4 & 0.025 & $(0.023-0.029)$ \\
\hline Vancomycin PO & 16 & 0.028 & $(0.011-0.062)$ \\
\hline
\end{tabular}

At the study centre, the long-term care units that might benefit most from antimicrobial stewardship were the physical support and cognitive support units, since these had the highest numbers of antibiotic orders per patient encounter. Clostridium difficile infections were treated for a mean duration of 10 days with oral or IV metronidazole or oral vancomycin, which are appropriate choices, according to guidelines of the Society for Healthcare Epidemiology of America and the Infectious Diseases Society of America. ${ }^{15}$

The limitations of this study include aspects of the design (retrospective, single-centre, descriptive); the limited study period (1 year); the predominantly male patient population; affiliation of the long-term care facility with a teaching hospital and all of its resources; and the absence of certainty in linking specific culture and susceptibility results with antibiotic use, presumed diagnosis, or appropriateness of antibiotic dosing (based on the temporal sequence of testing and prescribing). Despite these limitations, this study has provided useful information supporting further prospective evaluation of the need for antimicrobial stewardship in long-term care facilities in certain key areas, namely targeting cephalosporin and fluoroquinolone use and targeting prolonged duration of therapy (longer than 7 days). On the basis of the current findings, we suggest that prospective audit and feedback of frequently used antibiotic classes (third-generation cephalosporins and fluoroquinolones) and of all patients who have been taking antibiotics for longer than 7 days may be initiatives that could affect the care of patients in long-term care facilities and thus warrant evaluation. This study has shown that a long-term care facility affiliated with a teaching hospital and all of its resources nonetheless faces challenges similar to those associated with antimicrobial use in community facilities. In addition, this study used a methodology that other long-term care facilities could employ to assess their own antibiotic use. 
Implementation and evaluation of antimicrobial stewardship initiatives targeting one or more of the identified areas of concern listed above are planned at the study facility, with a goal of sharing findings in a future publication.

\section{CONCLUSION}

Characteristics of antimicrobial use at this long-term care facility that might benefit from further evaluation include potentially excessive use of fluoroquinolones and cephalosporins and potentially excessive duration of antibiotic use for individual patients.

\section{References}

1. Strausbaugh LJ, Joseph CL. The burden of infection in long-term care. Infect Control Hosp Epidemiol. 2000;21(10):674-9.

2. Dellit TH, Owens RC, McGowan JE Jr, Gerding DN, Weinstein RA, Burke JP, et al. Infectious Diseases Society of America and the Society for Healthcare Epidemiology of America guidelines for developing an institutional program to enhance antimicrobial stewardship. Clin Infect Dis. 2007;44(2):159-77.

3. Hecker MT, Aron DC, Patel NP, Lehmann MK, Donskey CJ. Unnecessary use of antimicrobials in hospitalized patients: current patterns of misuse with an emphasis on the antianaerobic spectrum of activity. Arch Intern Med. 2003;163(8):972-8.

4. Nicolle LE, Bentley DW, Garibaldi R, Neuhaus EG, Smith PW. Antimicrobial use in long-term-care facilities. SHEA Long-Term-Care Committee. Infect Control Hosp Epidemiol. 2000;21(8):537-45.

5. Hughes JM. Preserving the lifesaving power of antimicrobial agents. JAMA. 2011;305(10):1027-8.

6. Boucher HW, Talbot GH, Benjamin DK Jr, Guidos RJ, Jones RN, Murray BE, et al. $10 \times$ '20 progress - development of new drugs active against gramnegative bacilli: an update from the Infectious Diseases Society of America. Clin Infect Dis. 2013;56(12):1685-94.

7. Spellberg B, Guidos R, Gilbert D, Bradley J, Boucher HW, Scheld WM, et al. The epidemic of antibiotic-resistant infections: a call to action for the medical community from the Infectious Diseases Society of America. Clin Infect Dis. 2008;46(2):155-64.

8. Kaki R, Elligsen M, Walker S, Simor A, Palmay L, Daneman N. Impact of antimicrobial stewardship in critical care: a systematic review. J Antimicrob Chemother. 2011;66(6):1223-30.

9. Elligsen M, Walker SA, Pinto R, Simor A, Mubareka S, Rachlis A, et al. Audit and feedback to reduce broad-spectrum antibiotic use among intensive care unit patients: a controlled interrupted time series analysis. Infect Control Hosp Epidemiol. 2012;33(4):354-61.

10. Palmay L, Elligsen M, Walker SAN, Pinto R, Walker S, Einarson T, et al. Hospital-wide rollout of antimicrobial stewardship: a stepped-wedge randomized trial. Clin Infect Dis. 2014;59(6):867-74.

11. Daneman N, Gruneir A, Newman A, Fischer HD, Bronskill SE, Rochon PA, et al. Antibiotic use in long-term care facilities. J Antimicrob Chemother. 2011;66(12):2856-63.
12. Jump RLP, Olds DM, Seifi N, Kypriotakis G, Jury LA, Peron EP, et al. Effective antimicrobial stewardship in a long-term care facility through an infectious disease consultation service: keeping a LID on antibiotic use. Infect Control Hosp Epidemiol. 2012;33(12):1185-92.

13. Veterans \& Community: about our program [website]. Toronto (ON): Sunnybrook Health Sciences Centre; [cited 2013 Mar 19]. Available from: www.sunnybrook.ca/content/?page=veterans_centre_community

14. Elligsen M, Walker SAN, Simor A, Daneman N. Prospective audit and feedback of antimicrobial stewardship in critical care: program implementation, experience, and challenges. Can J Hosp Pharm. 2012;65(1):31-6.

15. Cohen SH, Gerding DN, Johnson S, Kelly CP, Loo VG, McDonald LC, et al. Clinical practice guidelines for Clostridium difficile infection in adults: 2010 update by the Society for Healthcare Epidemiology of America (SHEA) and the Infectious Diseases Society of America (IDSA). Infect Control Hosp Epidemiol. 2010;31(5):431-55.

Lisa Dong-Ying $\mathbf{W u}, \mathrm{BScPhm}$, was, at the time of this study, a pharmacy research student in the Department of Pharmacy, Sunnybrook Health Sciences Centre, Toronto, Ontario. She is now with the Department of Pharmacy at West Park Healthcare Centre, Toronto, Ontario.

Sandra A N Walker, BSC, BSCPhm, PharmD, FCSHP, is with the Department of Pharmacy, Sunnybrook Health Sciences Centre, Toronto, Ontario. She has affiliate status with the Department of Medicine, Division of Infectious Diseases, and Division of Clinical Pharmacology, Sunnybrook Health Sciences Centre; the Sunnybrook Research Institute; and the Leslie L Dan Faculty of Pharmacy, University of Toronto.

Marion Elligsen, BScPhm, is with the Department of Pharmacy, Sunnybrook Health Sciences Centre, Toronto, Ontario.

Lesley Palmay, BSc, BScPhm, MSc, is with the Department of Pharmacy, Sunnybrook Health Sciences Centre, Toronto, Ontario.

Andrew Simor, MD, is with the Department of Microbiology and the Division of Infectious Diseases, Sunnybrook Health Sciences Centre; the Sunnybrook Research Institute; and the Faculty of Medicine, University of Toronto, Toronto, Ontario.

Nick Daneman, MD, MSc, is with the Division of Infectious Diseases, Sunnybrook Health Sciences Centre; the Sunnybrook Research Institute; the Faculty of Medicine, University of Toronto; and the Institute for Clinical Evaluative Sciences, Toronto, Ontario.

Competing interests: None declared.

Address correspondence to:

Dr Sandra A N Walker

Department of Pharmacy

E-302, Sunnybrook Health Sciences Centre

2075 Bayview Avenue

Toronto ON M4N 3M5

e-mail: sandra.walker@sunnybrook.ca

Funding: None received. 